\title{
Cover illustration
}

\section{With a little help from my friends...}

$\mathrm{T}$ he rusty glow of a blooming marine phytoplankton, Oxcillatoria erythraea, can be startling when an entire bay is covered by what is commonly known as the "red tide." More subtle bluish bioluminescence can be seen with the gentle disturbance of the ocean surface colonised by the phytoplankton, Noctiluca. But sometimes, pelagic bioluminescence can be more than merely curious. Certain pelagic animals are known to cooperate with prokaryotes to use this ethereal light for defensive purposes.

The Pacific bobtail squid, Euprymna scolopes, is born having an empty bilobed sac within the centre of its mantle cavity. Shortly after the squid hatches, its sac is colonised by a Gram negative rod, Vibrio fischeri, a luminous bacterial symbiont selected by the squid for this purpose. $V$ fischeri is the only bacterial species capable of colonising a normal light organ in this squid. Ciliated cells on the ventral surface of the squid beat, passing seawater over and into the pores of this organ. Apparently, the ciliated epithelial cells produce mucus that can be used to "farm" the seawater of $V$ fischeri (McFall-Ngai MJ, Comp Bio Physiol part A 2000;126:471-80). As the bacteria colonise the crypts of this organ, they multiply rapidly and within 12 hours, the initial inoculum has risen to $10^{6}$ or even $10^{9}$ in adults, and this combination results in a 1000 -fold increase in the luminescence from the organ. But, this dramatic increase in luminescence is not entirely caused by the bacteria as the squid can manipulate the crypts in the light organ, changing the oxygen concentration to influence bioluminesence. Once initial colonisation occurs the ciliated epithelial cells that were key to the colonisation are sloughed and entirely lost.

Once $V$ fischeri has been established in the light organ, a repetitive cycle begins. Each morning, the squid discharges approximately $95 \%$ of the bacteria, leaving the most vigorous and adapted $V$ fischeri. The squid burrows into pebbles and broken coral on the bottom and waits. During this time the bacteria enjoy rapid doubling and 12 hours later will have the aforementioned 1000-fold increase in luminescence.

This bilobed sac, or light organ, within the mantle of the squid is

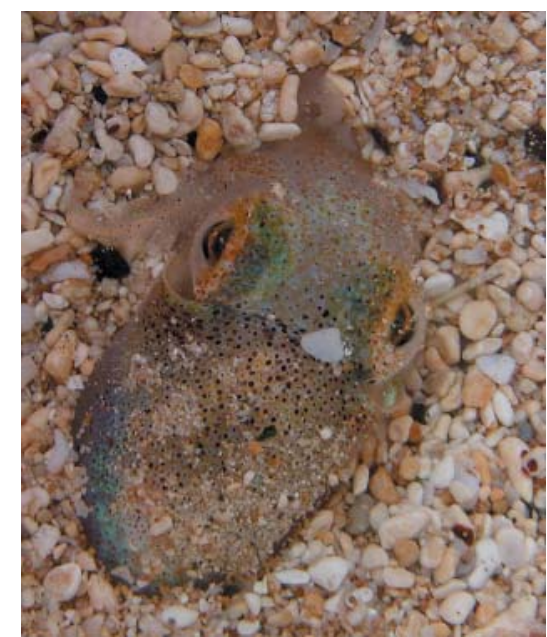

the tapetal mechanisms in some animals (BJO cover essay, May 2005). E scolopes has these proteins scattered across its body, including in its eye, skin, and digestive gland, the liver analogue. The nanofabrication of these proteins is unique to cephalopods and is specifically different from the more common aquatic reflective tissue such as purine or guanine.

Such protective mechanisms must have been successful because the squid is widespread and common in its range. $E$ scolopes may even use this sub-mantle flashlight like a torch illuminating its prey species from above and in this regard is reminiscent of $M$ niger (BJO cover essay, March 2004)

This remarkable symbiotic relationNote small pebbles used as camouflage. See BJO cover essay, Jan 2001 for description and explanation of integument colour change.

covered dorsally by the ink sac to limit stray light radiating beyond the organ. The organ itself is lined with a newly described protein, reflectin, that directs the light to be emitted ventrally. Furthermore, there is a formed ventral lens, derived from muscle, with lenticular proteins very similar to the lateral eyes, to further concentrate, and focus, the light ventrally.

$E$ scolopes uses this light organ in defence to mimic the downwelling light from above. It might not seem that light production could be used in defence as camouflage, but it unquestionably is. As the squid hunts for its favourite prey, shrimp, it provides a darkened silhouette to predatory fish below it as it blocks out the light from the moon or stars above. But when it replicates the downwelling light even to the appropriate wavelength, the predator cannot distinguish the squid from moonlight. Of course, some night skies would be brighter than others and so the squid controls its light emission with a biological diaphragm, replicating the quantity of downwelling light it produces. This is done with the ink sac which can provide ink for defence as well, but otherwise controls the amount of light emitted (Jones et al, Marine Biology 2004; 144:1151-5).

The reflectins are unique proteins that work by thin film interference much like ship is representative of the symbiotic mutualism most metazoans have and often require. Colonisation of the gut and skin of metazoans by prokaryotes is very common, and may be commensal or symbiotic. Consortia of prokaryotes and metazoa are probably very old and may have had a role in the radiation of metazoa. Sometimes individual symbionts are not capable of living alone or without one another. Arguably the most extreme example of such symbiotic mutualism and simultaneous endosymbiosis arguably is the mitochondrial-eukaryotic cell relationship. It is that marriage that gives us mitochrondria.

Perhaps the most intriguing aspect of this infection of the $E$ scolopes light organ is this. $V$ fischeri is closely related to $V$ cholerae, which can be a pathological organism. What enables the squid to maintain such an organism without overgrowth or internal damage? Since there are organisms that are pathological to some species and symbionts to others, there may be much to learn from such co-evolved interaction.

The squid co-opts this light as both a defence and possibly as an agent of illumination through a remarkable symbiotic relationship. All this is done with just a little help from its friends.

\section{R Schwab \\ University of California, Davis, Sacramento, CA, USA; irschwab@ucdavis.edu}

Photographs by Margaret McFall-Ngai. Thanks to her for review of essay. 